\title{
Developing Schizophrenia
}

John Cromby

In press 'Theory \& Psychology'

John Cromby

School of Management

Leicester University

University Road, Leicester

LE1 7RH England UK

john.cromby@leicester.ac.uk 


\title{
Developing Schizophrenia
}

\begin{abstract}
Neuroscience and schizophrenia are densely entangled and mutually supporting, such that a critical evaluation of schizophrenia is, effectively, an evaluation of applied aspects of contemporary neuroscience. A critical historical account of the development of schizophrenia is therefore followed by an overview of current issues and debates. A summary of possible future research directions then identifies a range of extant research strategies which already undercut or exceed this diagnosis. It is concluded that the example of schizophrenia functions more generally to illustrate how neuroscience need not be reliant upon poorly supported psychiatric concepts of mental illness.
\end{abstract}

\section{Introduction}

There are multiple, reciprocal and mobile relations between neuroscience, persons and their brains, and psychiatric diagnoses such as schizophrenia. Understandings of schizophrenia characteristically implicate notions of the brain, its normal workings, and its potentials to go functionally or structurally awry. Research in neuroscience is frequently justified with respect to its claimed potential to lead to new interventions for diagnoses such as schizophrenia. Significant research funding in the biosciences generally, including the neurosciences, has historically flowed from pharmaceutical companies seeking to develop and test new drug treatments for schizophrenia and related diagnoses (Rose \& Rose, 2012). These treatments have sometimes given rise to hypotheses about brain function in schizophrenia (and other psychiatric diagnoses), hypotheses which have proliferated throughout neuroscience more generally. Conversely, neuroscientific research into the structure and functioning of healthy brains continues to inform the development of new hypotheses about impaired structure or unhealthy function with respect to schizophrenia and related diagnoses. These developments also get taken up within other biosciences, such as epigenetics, and within cognate disciplines including psychology.

In short, neuroscience and schizophrenia are closely linked in a dense, interdependent, evolving nexus of ideas, practices, technologies and knowledge. Conceptualisations of mental health and illness, concepts and images of brains, their parts and their functions, practices of treatment and intervention, and the disparate interests of multiple professions - most proximally, neuroscience, psychiatry, psychology, pharmacology - are continuously circulated and exchanged, and mutually, dynamically and contingently associated. If not actually symbiotic, then, neuroscience and psychiatry are certainly inter-related and mutually-informing, to such an extent that Insel \& Quirion (2005) could describe psychiatry as 'clinically applied neuroscience'.

A critical examination of schizophrenia and (some of) the research associated with it is therefore one way of illuminating neuroscience more generally. Contemporary neuroscience is, largely inadvertently, challenging and deconstructing psychiatric diagnosis by demonstrating that there are no distinct neural signatures corresponding to functional diagnoses such as schizophrenia.

Simultaneously, it is accelerating tendencies toward the molecularisation of social science and psychology (that is, the reduction of diverse social influences to the presumed 'final chemical pathways' by which they impact upon biological systems), and so fostering the illusion that the causes and consequences of psychological distress can be understood wholly in neural terms. A critical analysis of schizophrenia must therefore recognise that the dense traffic between psychology, psychiatry and neuroscience has both progressive and regressive potentials. 
Before presenting any such analysis it is necessary to emphasise that, whilst, schizophrenia remains a contested concept, the overwhelming majority of critics today recognise that the experiences associated with this diagnosis are typically traumatising, debilitating, and associated with marked occupational and social dysfunction. Unlike some high-profile work associated with the so-called anti-psychiatry movement, contemporary critiques rarely try to normalise the experiences associated with this diagnosis (although they may note that voice-hearing, for example, is in fact quite prevalent in the general population - Beavan, Read, \& Cartwright, 2011). Nor do they position schizophrenia as something heroic (cf. Deleuze \& Guattari, 1984), albeit that they do recognise that voice-hearing and related experiences can have positive aspects (Romme, Escher, Dillon, Corstens, \& Morris, 2009). Whatever the scientific status of schizophrenia, it is recognised that those given the diagnosis are frequently in urgent need of help and that it's worldwide diagnostic prevalence of around $1 \%$ indexes significant social, economic and personal costs. Researchers, whether accepting of the schizophrenia concept or critical of it, are therefore mostly united in the goal of clarifying the causes, character and consequences of the experiences associated with a diagnosis, and so developing more effective remedial and preventative measures. It follows that the aim of critique is certainly not to negate the relevance of neuroscience: rather, to clarify and develop its contribution in order to maximise its potential benefits. With this aim in mind, I will consider some implications for and connections to neuroscience of three aspects of schizophrenia: it's initial development, it's current status, and it's likely future development.

\section{Initial Development}

This section draws primarily upon Boyle's (2002) critical history of schizophrenia. This is appropriate for three reasons: first, Boyle presents a particularly close reading of original writings by Kraepelin, Bleuler and others who developed the concept; second, she is a clinical psychologist so her analysis (which is not solely historical) is informed by clinical practice; and third, whilst influential and highly regarded in UK clinical psychology, Boyle's work is less well-known elsewhere. Like Heinrichs (2003), Boyle's history recognises that schizophrenia emerged relatively suddenly early in the $19^{\text {th }}$ century, and like Berrios, Luque \& Villagran (2003) she rejects the 'continuity hypothesis' that schizophrenia has always existed and that work during the last century has identified an ontologically real, stable and unitary disease entity. Accordingly, like Berrios et al., Boyle engages with, rather than glosses over, differences between the early architects of this putative disease, and between these early works and the concept as it exists today.

Most histories trace schizophrenia to the publication of Kraepelin's 1896 research on what he called dementia praecox. This term, meaning 'early dementia', was occasionally used as early as the 1850 's, and it is also sometimes said that the disease was first medically described in case studies dating from 1797 and 1809 (Heinrichs, 2003). Nonetheless, Kraepelin's work, which claimed to distinguish dementia praecox from other putative diseases of asylum inmates, is typically identified as the origin of the modern concept. Exemplifying the biologically-oriented German psychiatric tradition, Kraepelin's account characterised dementia praecox as a whole-body disorder, which he hypothesised was produced by systemic, metabolic or 'auto-intoxicating' disease processes that cascaded through the endocrine and peripheral nervous systems until, eventually, they reached the brain (Noll, 2011).

It was Bleuler who in his 1911 work deployed the term schizophrenia, although he was clear that by this he meant the same disorder that Kraepelin called dementia praecox. Bleuler's new term 
reflected his observation that - unlike in dementia - outcomes were sometimes positive, and also mirrored his own conceptualisation of the disease. Whereas Kraepelin largely emphasised biology, Bleuler (partly due to the influence of Freud, an influence often mediated by Bleuler's research assistant, Jung - Makari, 2008) proposed a more diverse theorisation - albeit one with biology at its root. Bleuler proposed that an (unspecified) brain impairment produced a cognitive dysfunction which, in turn, disrupted the usual seamless integration of psychological functions such as memory, affect and self-awareness. This disruption interfered with typical processes of association and inhibition, allowing excesses of affect to form and to become associated with memories and symbols, creating what Bleuler called 'idea complexes'. Whilst these idea complexes are the basis of the delusions and hallucinations commonly said to characterise schizophrenia, in Bleuler's concept these dramatic, disturbing experiences are merely secondary effects of a brain disease which is first of all organic and then cognitive.

The concept of schizophrenia subsequently underwent further revision by Schneider in 1956, and has since been modified less substantially in each revision of the main psychiatric diagnostic manuals, the International Classification of Diseases (ICD) and the Diagnostic and Statistical Manual of the American Psychiatric Association (DSM). Most revisions are made with the explicit goal of improving diagnostic reliability: for example, when DSM5 was released in 2013 it abolished the five sub-categories of schizophrenia (hebephrenic, catatonic, paranoid, disorganised and undifferentiated) introduced in DSMIII. Nevertheless, for the most part psychiatry continues to trace the history of schizophrenia jointly back to Kraepelin and Bleuler, uniting their two concepts in a foundational origin story that posits the discovery and elucidation of a new disease (e.g. Burton, 2006; Fusar-Poli \& Politi, 2008). Kraepelin and Bleuler are described as "founding fathers" who first supplied the notion that, whilst schizophrenia has many different manifestations, it is in essence "a disease of the mind that is characterised by a 'disturbance in thinking' - an abnormality in a fundamental cognitive process" (Andreasen 2000, p 108). So despite modifications to diagnostic criteria, and notwithstanding occasional challenges such as those posed by the so-called antipsychiatry movement, the psychiatric conception of schizophrenia as an organic brain disease, that gives rise to a cognitive dysfunction which fragments experience and thought, continues to predominate.

Boyle's (2002) historical analysis recognises the foundational status afforded to Kraepelin and Bleuler. She presents a comprehensive analysis of their writings, framed by a history of some of the significant social changes of the $18^{\text {th }}$ and $19^{\text {th }}$ centuries, including the rise of the asylum, the birth and decline of moral management, and the relations between neurology and the nascent field of psychiatry as they appeared in different countries, particularly Germany (where they were especially closely related). Since Boyle's lengthy account defies summary within the confines of a single paper, this précis will emphasise her reading of Kraepelin. One of Boyle's key contentions, which she evidences by a close reading of Kraepelin's works, is that he:

"in fact appears to have been unaware of, or to have chosen to ignore, even the most basic principles of empirical enquiry - the need to present systematically gathered data, rather than to rely on personal experience and beliefs; the importance of clear description so that others can try to replicate the observations; the importance of reliability of observations and the dangers of question begging." (Boyle 2002, p.59) 
The significance of this contention reflects the foundational status of Kraepelin's work (including its uptake by Bleuler). If, as Boyle, suggests, the initial processes whereby the concept of dementia praecox was derived was inadequate, we must question subsequent work elaborating, refining, and seeking causes and treatments for it: just as we might question the validity of work refining personality structures based upon astrological star signs, since the existence of distinct personalities consistently linked to birth dates has never been convincingly demonstrated.

Boyle first of all engages with Kraepelin's early work, in which he claimed to be using observed similarities in onset, course and outcome to identify dementia praecox amongst asylum inmates. She observes that whilst each of these is actually an umbrella term covering a range of continuous changes and dynamic processes, they might nevertheless be deployed after a coherent disease construct had already been identified. However, Kraepelin's use of them to initially identify dementia praecox was problematic, since it was logically impossible for him to know in advance of their identification which of the (typically many) observed similarities in onset, course and outcome were significant and related. These difficulties were further compounded because his judgements of onset and (to some extent) course necessarily relied upon retrospective data, which may or may not have recorded changes whose putative significance would only later become apparent, in relation to the new construct. A somewhat different problem arose in respect of outcome, since nothing definitive could be concluded under this heading until no more changes were actually able to occur.

Given the intransigence of these problems, Boyle finds it unsurprising that Kraepelin described difficulties using the criteria of onset and course:

"The whole upheaval can take so imperceptibly and with such indefinite indications that those around imagine that they are confronted simply with the outcome of an unhappy development, perhaps even of some character fault"

"In more than half the cases, the upheaval occurs so imperceptibly and with such indefinite indications that its actual beginning cannot be determined in retrospect."

"The course of this process of illness can take the most varied forms"

"The further course of the illness in these cases is a varied one insofar as the imbecility sometimes develops more rapidly, sometimes more slowly, and can in fact stop progressing at very different stages"

Kraepelin, in Boyle (2002, p.47-49)

Likewise, in relation to the criterion of outcome Kraepelin provided only what Boyle calls 'highly varied' descriptions with no indications of actual numbers of inmates to whom they applied. These descriptions were accompanied by what she calls 'vague statements' about psychological characteristics, statements neither linked systematically to observations nor supported by numerical analyses:

"although patients are more placid, it is only to reveal ever more clearly the indications of a fairly high-grade psychological weakness"

"The common outcome of all severer forms of dementia praecox is idiocy" 
"Most frequently, however, the illness seems to lead to an insane confusion"

Kraepelin, in Boyle (2002, p.50-51)

Consequently, Boyle questions the validity and reliability of the criteria Kraepelin used to initially identify dementia praecox. Vitally, she further observes that Kraepelin did not only need reliable and valid criteria: he also needed to present systematic analyses of the within-group similarities and between-group differences these criteria produced. Boyle finds, however, that nowhere did Kraepelin present any such systematic analyses. She suggests that his writings obviate this need by deploying phrases such as "'one often notices'; 'it is occasionally observed'; 'in some cases'" (Boyle 2002, p.46): phrases which seemingly ground his work in thorough observation without ever reporting actual numbers of inmates who conformed - or not - to the descriptions he presented.

Boyle therefore concludes that Kraepelin must have effectively been working backwards: rather than present, enumerate and analyse evidence on the basis of systematic observation, and then infer the existence of a new disease construct on that basis, Kraepelin seemingly began with some idea of the construct and then proceeded to describe what he said were cases of it. So despite the wealth of detailed observations Kraepelin presents, the process by which he analysed them to derive a putative new brain disease was inadequate. Rather than conduct the necessary systematic analyses of these (in any case problematic) observations, using within/between-group comparisons to derive robust differences, Kraepelin seems to have performed an impressionistic reading that would, inevitably, have tended to confirm what he already believed.

Bleuler then took Kraepelin's claims to have discovered a new disease largely on trust, just as, subsequently, Schneider accepted the work of both of his predecessors largely uncritically (and just as the DSM and ICD later did with the work of all three men). Boyle finds this especially remarkable given that the symptom profiles presented by both Kraepelin and Bleuler as characteristic of schizophrenia consistently included a striking range of neurological and physiological problems tremors, tics, paralyses, gait disorders, oedemas, excessive sweating and cyanosis of the hands and feet, as well as delusions and hallucinations - that are rarely, if ever, seen amongst today's patients. Boyle suggests that (whilst it is impossible to be certain) this might be because many of Kraepelin and Bleuler's patients were actually suffering from undiagnosed encephalitis lethargica - a viral disease first identified by von Economo in 1917, the symptoms of which include all of those identified by Kraepelin and Bleuler as symptoms of schizophrenia. It is now known (but could not have been known by Kraepelin or Bleuler) that there were recurrent epidemics of encephalitis lethargica in Europe during the late 1800's and early 1900's, so significant numbers of asylum inmates may well have been suffering with its effects. Whilst the extent to which Kraepelin and Bleuler took cases of this neurological disorder as cases of schizophrenia/dementia praecox is impossible now to decide, their doing so would account for the otherwise puzzling change that has occurred in the typical symptoms they identified and those that typify schizophrenia today.

As a clinician, Boyle's aim is neither to dismiss nor normalise the experiences associated with schizophrenia, but to clarify their nature and causes so that more effective interventions can be tailored. In the present context, her analysis speaks directly to what Cohn (2010) characterises as a paradox in the intimate relationship between psychiatry and neuroscience. On the one hand, the reliability and validity of neuroscientific research into schizophrenia largely depends upon the adequacy and stability of the diagnosis. On the other hand, this dependency co-exists alongside a 
more general view, often expressed relatively obliquely, that neuroscience must eventually develop its own ways of establishing pathology that are independent of such categories. Consequently, neuroscientific research in schizophrenia holds that biological diseases or impairments are the necessary basis of the experiences associated with this diagnosis, whilst simultaneously striving to identify abnormalities that underlie or exceed, rather than simply reinforce, this and other psychiatric diagnoses.

Clearly, if Kraepelin and Bleuler never did establish schizophrenia as a valid disease concept, the neuroscientific paradox that Cohn identifies becomes considerably sharper, and the (sometimes covert) difficulties it generates for neuroscientists are potentially more widespread. Moreover, as consideration of the current status of schizophrenia shows, it is at least possible that neuroscience is already encountering such difficulties.

\section{Current Status}

So schizophrenia has from the earliest been framed as a brain disease or dysfunction - albeit one with an unspecified pathology. This neural emphasis is demonstrated by a bibliometric analysis of abstracts of nearly 10,000 papers presented at two major international conferences on schizophrenia between 1988 and 2004 (Calton, Cheetham, D'Silva, \& Glazebrook, 2005), which found that $75 \%$ were primarily biological in their orientation, whereas less than $5 \%$ took a predominantly psychosocial perspective (and less than $2 \%$ gave explicit consideration to actual experiences). With respect to neuroscience schizophrenia is thus positioned as a highly fertile concept, one that seemingly yields unanswered questions, the possibility of fame and even fortune, and the opportunity to make a significant difference to the quantum of human suffering. At the same time, Rose \& Abi-Rachid (2013) show that psychiatry has long struggled to define the boundaries of sanity, madness and normality, and observe that with the rise of community treatment in recent decades the boundaries between neurosis and psychosis have become more blurred. Hence, statements about the interpretation of diagnostic criteria increasingly emphasise heterogeneity within the categories, even whilst at the same time promoting degrees of specificity in their presumed underlying biological pathologies (for example, in psychiatry's continuing idea of schizophrenia as an organic disease of higher cognitive processes). There are enduring problems of validity and reliability associated with all of the functional psychiatric diagnoses, schizophrenia included (Johnstone, 2001), which means that the continuously-evolving relationships between psychiatry, schizophrenia and contemporary neuroscience, whilst mutually imbricative, are necessarily complex and sometimes contradictory.

Rose \& Abi-Rachid (2013) also observe that neuroscience has frequently mobilised and traded in the concept of schizophrenia, just as psychiatry has exploited the cachet and resources of neuroscience to warrant its conceptual frameworks and promote its own research agendas. Nevertheless, overall progress has been disappointing. Despite some claims to have found 'the' neural or genetic basis of schizophrenia, and notwithstanding that efforts to do so have benefitted from very generous research funding and, in recent years, an explosion of powerful new technologies, the results of this massive research effort remain largely inconclusive. Cromby, Harper, \& Reavey (2013) observe that in recent decades schizophrenia has been associated with abnormalities of or differential functioning within dopamine, glutamate, serotonin, acetylcholine, gamma-butyric acid, prostaglandin and neuropeptide systems and pathways. At the same time, schizophrenia has also been associated with neuroanatomical features including enlarged ventricles, cerebral asymmetry, temporal lobe 
abnormalities, thickened corpus callosum, thinner corpus callosum, abnormalities of the basal ganglia and cerebellum, and reduced overall brain volume. So it is not that there is no evidence of neural variation in relation to the diagnosis of schizophrenia: rather that there is no coherent pattern of evidence consistent with claims of a distinct neural pathology as the basis of this diagnosis. These problems are compounded by others, notably that the results of many studies are confounded by the effects of psychiatric medication; and also that both population norms and what Steven Rose (1997), following Dobzhansky, calls 'norms of reaction' (i.e. the range within which phenotypic gene expression can vary without functional failure, and beyond which usual functioning breaks down) are largely unavailable for these various features: hence neither their pathological significance, nor their prevalence in the general population, have typically been definitively established. Hence, some fifteen years ago an editorial in Nature Neuroscience $(1999$, p.295) noted that:

"Schizophrenia remains unexplained. None of the abnormalities reported in the brains of schizophrenics is clearly diagnostic for the disease in the way that (say) plaques and tangles are for Alzheimer's disease."

And this uncertainty continues unabated:

"the field of psychiatry has thus far failed to identify a single neurobiological phenotypic marker or gene that is useful in making a diagnosis of a major psychiatric disorder" (Charney et al. 2002, p.33)

"Our understanding of the biological mechanisms of diseases such as mood disorders, schizophrenia and autism is frustratingly limited .. There is also a lack of reliable biological markers for characterising these diseases". (Chou \& Chouard 2008, p.889)

"efforts to understand the neurobiological bases of the clinical heterogeneity that schizophrenia comprises, mainly by correlating neurobiological measures with specific symptoms, have been largely unsuccessful. Indeed, it is fair to say that "inconsistency" has been the most consistent finding to emerge from such efforts." (Mathalon \& Ford, 2012)

Whilst genetic research is not the focus here, it should be noted that these acknowledgements also reflect recent molecular genetic findings in schizophrenia, which have failed to discover any major genes of significant effect associated with this diagnosis (Crow, 2008). Consequently, although the notion that schizophrenia is a brain disease continues to predominate, in neuroscience and in psychiatry, current research is developing in strikingly diverse ways. With respect to its neural and biological aspects this diversity can be illustrated by considering a recent issue of the leading journal 'Schizophrenia Bulletin', which in November 2013 published papers relating schizophrenia to endophenotypes identified by P50/P300 ERP events; variability in the ZNF804A gene; disruption of corollary discharge function for motor movements; temporal lobe volume abnormalities; aberrant salience and dopamine activity; low birth weight; connectivity between the default mode network and task-processing networks after ingesting psilocybin; and activation differences amongst people given the diagnosis of schizophrenia in the posterior cingulate, precuneus and other regions in selfother differentiation tasks. These various strands of investigation have many affinities with work in (predominantly) cognitive and affective neuroscience (and in genetics and epigenetics), and it remains hypothetically possible that one might ultimately identify a distinct neural pathology for schizophrenia. Nevertheless, whilst it remains highly prevalent, promissory discourse of this kind is 
now increasingly accompanied by other discourses associated with somewhat different research strategies.

\section{Possible Futures}

Rather than simply continue searching for brain impairments specific to and consistently associated with schizophrenia, many researchers are now adopting alternative strategies. As Cohn (2010) suggests, for some neuroscientists the problem is primarily the inadequacy of diagnosis: hence, anticipating publication of DSM5, the director of the American NIMH Thomas Insel announced in April 2013 that the Institute's future funding strategy would not rely upon psychiatric diagnostic criteria. Whilst this statement was later qualified, the new NIMH strategy - of developing its own Research Domain Criteria as an alternative, more valid taxonomy of distress - remains. In this regard, it has been argued that philosophical phenomenology might contribute to neuroscientific research by 'front-loading' studies with consistently established experiential distinctions which might, in turn, assist in the identification of related neural systems (Woods et al., 2014). Another strategy that has long been evident is to conceive of schizophrenia as a syndrome: to figure it within diagnostic manuals as a singular disease, whilst simultaneously describing it elsewhere as a cluster of related conditions (e.g. Roberts, 1990). Conversely, Frangou (2014) advocates a strategy of using imaging technologies within a 'systems neuroscience' perspective with respect to both schizophrenia and bipolar disorder. Although here (as in other recent studies e.g. Fillman, Sinclair, Fung, Webster, \& Shannon Weickert, 2014) both diagnoses can be subsumed within the broad category of psychosis, other diagnostic reconfigurations are also appearing: for example, a meta-analysis by the Cross Disorder Group of the Psychiatric Genetics Consortium (2013) claimed to identify putative genetic influences operating equally with respect to diagnoses of schizophrenia, bipolar disorder, major depression, autism and ADHD. An alternate strategy involves leaving diagnosis largely unquestioned but adopting different bioscientific research strategies: Light \& Swerdlow (2014) propose using biomarkers to identify unimpaired neural and cognitive function amongst people given a schizophrenia diagnosis; using drug challenges and practice effects to identify areas of continuing neuroplasticity; and monitoring therapeutic progress in these areas using neurophysiological measures such as prepulse inhibition of startle.

In recent years there has also been a resurgence of interest in the social and relational influences associated with schizophrenia. There is robust evidence showing, for example, that social inequality and low SES are causally associated with a schizophrenia diagnosis (Harrison, Gunnell, Glazebrook, Page, \& Kwiecinski, 2001); that ethnic minority status and the effects of discrimination and prejudice make schizophrenia diagnoses significantly more likely (Boydell et al., 2001); and that child physical and sexual abuse, neglect, bullying and emotional abuse are all causal of the experiences associated with a schizophrenia diagnosis (Read, van Os, Morrison, \& Ross, 2005). Relevant research in neuroscience (and other biosciences, notably epigenetics) consequently aims to identify specific neural pathways or processes modified by these toxic combinations of adverse circumstances (e.g. Tyrka, Price, Marsit, Walters, \& Carpenter, 2012)

Another range of alternative strategies are emerging from (predominantly British) clinical psychology. Inspired in part by Boyle's work, many researchers and practitioners largely disregard the concept of schizophrenia (Bentall, 2003). It is not that they necessarily overtly reject the diagnosis (although their professional body has done so-DCP, 2013), rather that they do not invoke it as a causal explanation. Research (and interventions) associated with this perspective focus on 
specific, relatively homogenous experiences - hearing abusive voices, holding unusual and distressing beliefs - rather than relatively heterogeneous diagnostic categories, and uses experimental techniques to identify cognitive, affective, neural and physiological processes associated with these more specific difficulties. For example, Bentall, Kinderman, \& Kaney (1994) found that a combination of low self-esteem and external locus of control were associated with the presence of persecutory delusions; they argued that self-worth was being defended by (mis-) attributing negative occurrences to malevolent others. Conversely, Garety et al. (2005) used measures of reasoning, emotion, belief inflexibility and extreme responding to demonstrate that people with delusional beliefs displayed evidence of the cognitive bias of 'jumping to conclusions'. Likewise, a meta-analysis of externalising biases in people experiencing auditory hallucinations (Brookwell, Bentall, \& Varese, 2013) found robust, moderate to large effects associating these experiences with cognitive impairments in source monitoring (i.e. attributing self-generated 'inner speech' to an external source).

Whilst there are methodological parallels between neuroscience and this clinical psychological research, substantive connections are sometimes less immediately apparent. Nevertheless, researchers acknowledge that neural systems continuously enable (albeit not simply cause - Harre, 2002) all psychological phenomena, and this provides the basis for collaborations and, in some cases, models that combine psychological and neuroscientific influences (Bentall, 2003). For example, Read's traumagenic neurodevelopmental model (Read, Fosse, Moskowitz, \& Perry, 2014) recasts the various discrepant brain features sometimes observed amongst people given psychoticspectrum diagnoses as injuries, caused by (predominantly early) trauma. The model embraces current neuroscientific evidence regarding brain development and the functions of different regions and systems, drawing upon imaging and related studies to demonstrate the many similarities between the brains of abused and neglected children and the brains of some people given diagnoses of schizophrenia. At the same time, by treating these brain features as injuries (not illnesses), and by focusing on the broader notion of psychosis rather than the more specific concept of schizophrenia, the model negates psychiatric conceptions of schizophrenia as brain disease.

\section{Conclusion}

Despite the foundational status accorded to Kraepelin and Bleuler's work, schizophrenia might not have been initially established as a coherent disease construct. This would help explain why subsequent research, instead of clarifying the basis of this presumed disease, has been largely inconclusive. If so, genuine progress toward identifying how neural systems enable the experiences associated with this diagnosis will only be made when neuroscientists abandon the schizophrenia concept. In any case, a range of neuroscience research strategies are already discernible, including:

- identifying biomarkers for unimpaired function

- identifying systemic neural deficits

- combining schizophrenia with other psychiatric diagnoses

- developing the NIMH Research Domain Criteria

- researching relatively homogenous experiences such as 'hearing voices'

- re-interpreting neural differences as injuries, rather than illnesses

Some of these research strategies are relatively orthodox in that they retain a notion of diagnosis and posit distinct causal neural pathologies; others are less so, dispensing with diagnosis and focusing instead on specific aspects of experience such as hearing voices. They differ in the extent to 
which they can readily incorporate the epidemiological evidence regarding trauma, abuse, SES and ethnicity; this is because illness-based strategies import a hierarchy of evidence within which neuroscientific or biological influences predominate, and only individuals with (presumed) biological deficits fall prey to symptoms. Consequently, they also differ in how they incorporate this epidemiological evidence - as the 'trigger' that might release a hidden neural vulnerability, or as the adverse circumstances that might cause brain injury. As a result, there is also considerable variation in the manner and extent to which these strategies open neuroscience to the social and cultural contexts within which it gets conducted (Choudhury \& Slaby, 2012). Nevertheless, to some degree each strategy recognises that the construct of schizophrenia does not provide a sufficiently sound basis for future neuroscientific research, and all offer viable alternatives that could generate rich empirical studies with considerable potential to expand knowledge and alleviate suffering.

The example of schizophrenia therefore shows that neuroscience need not confine itself to models of psychological functioning derived uncritically from biological psychiatry. Valid neuroscientific research programmes can be pursued without endorsing psychiatric diagnoses that lack reliability and validity, and indeed without presupposing medical notions of illness. Scholars who have recently suggested that social neuroscience could make a strong contribution to psychiatry (e.g. Caccioppo et al 2014) might want to pay particular heed to such suggestions. At the same time, they are relevant for neuroscience generally, precisely because it is so densely entangled with psychiatric theories, evidence and practice.

Considering its implications more broadly, this example also illustrates how neuroscience can benefit from a more sustained engagement with psychology and social science. Such research will encounter methodological, conceptual, linguistic and ideological problems (Cromby, 2007; Callard \& Papoulias 2010), and will raise challenges for psychology at the same time as it overturns established certainties in psychiatry. We should welcome these challenges and the opportunities they will bring.

\section{REFERENCES}

Andreasen, N. (2000). Schizophrenia: the fundamental questions. Brain Research Reviews, 31, 106112.

Beavan, V., Read, J., \& Cartwright, C. (2011). The prevalence of voice-hearers in the general population: a literature review. Journal of Mental Health, 20(3), 281-292.

Bentall, R. (2003). Madness Explained. London: Allen Lane / Penguin.

Bentall, R., Kinderman, P., \& Kaney, S. (1994). The self, attributional processes and abnormal beliefs: towards a model of persecutory delusions. Behaviour Research and Therapy, 32, 331-341.

Berrios, G., Luque, R. \& Villagran, J. (2003). Schizophrenia: a conceptual history. International Journal of Psychology and Psychological Therapy. 3,2: 111-140

Boydell, J., Van Os, J., McKenzie, K., Allardyce, J., Goel, R., McCreadie, G., et al. (2001). Incidence of schizophrenia in ethnic minorities in London: ecological study into interactions with environment. British Medical Journal, 323, 1336-1338.

Boyle, M. (2002). Schizophrenia: a scientific delusion? (2nd ed.). London: Routledge.

Brookwell, M. L., Bentall, R. P., \& Varese, F. (2013). Externalizing biases and hallucinations in sourcemonitoring, self-monitoring and signal detection studies: a meta-analytic review. Psychological Medicine, 43(12), 2465-2475.

Burton, N. (2006). Psychiatry. Oxford: Blackwells. 
Cacioppo, J., Cacioppo, S., Dulawa, S., \& Palmer, A. (2014). Social Neuroscience and its potential contribution to psychiatry. World Psychiatry, 13(2), 131-139.

Calton, T., Cheetham, A., D'Silva, K., \& Glazebrook, C. (2005). International Schizophrenia Research and the Concept of Patient-Centredness - an analysis over two decades. Paper presented at the International Congress on Schizophrenia Research, Savannah, GA, USA.

Charney, D., Barlow, D., Botteron, K., Cohen, J., Goldman, D., Gur, R., et al. (2002). Neuroscience Research Agenda to Guide Development of a Pathophysiologically Based Classification System. In D. Kupfer, M. First \& D. Regier (Eds.), A Research Agenda for DSM-V (pp. 31-84).

Chou, I. h., \& Chouard, T. (2008). Neuropsychiatric disease. [10.1038/455889a]. Nature, 455(7215), 889-889.

Choudhury, S., \& Slaby, J. (Eds.). (2012). Critical Neuroscience. Chichester: Wiley-Blackwell.

Cohn, S. (2010). Disrupting Images: neuroscientific representations in the lives of psychiatric patients. In S. Choudhury \& J. Slaby (Eds.), Critical Neuroscience: a handbook of the social and cultural contexts of neuroscience. Chichester: Wiley-Blackwell.

Cromby, J. (2007). Integrating Social Science with Neuroscience: potentials and problems. Biosocieties, 2(2), 149-170.

Cromby, J., Harper, D., \& Reavey, P. (2013). Psychology, Mental Health and Distress. London: Palgrave.

Cross Disorder Group of the Psychiatric Genetics Consortium. (2013). Identification of risk loci with shared effects on five major psychiatric disorders: a genome-wide analysis. Lancet. 381 (9875): 1371-1379

Crow, T. (2008). The emporors of the schizophrenia polygene have no clothes. Psychological Medicine, 38, 1681-1685.

DCP. (2013). Classification of behaviour and experience in relation to functional psychiatric diagnoses: Time for a paradigm shift. Leicester: British Psychological Society.

Deleuze, G., \& Guattari, F. (1984). Anti-Oedipus: capitalism and schizophrenia. London: Athlone Press.

Fillman, S. G., Sinclair, D., Fung, S. J., Webster, M. J., \& Shannon Weickert, C. (2014). Markers of inflammation and stress distinguish subsets of individuals with schizophrenia and bipolar disorder. [Original Article]. Transl Psychiatry, 4, e365.

Frangou, S. (2014). A Systems Neuroscience Perspective of Schizophrenia and Bipolar Disorder. Schizophrenia Bulletin.

Fusar-Poli, P., \& Politi, P. (2008). Paul Eugen Bleuler and the Birth of Schizophrenia (1908). American Journal of Psychiatry, 165(11), 1407.

Garety, P. A., Freeman, D., Jolley, S., Dunn, G., Bebbington, P., Fowler, D., et al. (2005). Reasoning, Emotions, and Delusional Conviction in Psychosis. Journal of Abnormal Psychology, 114(3), 373-384.

Harre, R. (2002). Cognitive Science: a philosophical introduction. London: Sage Publications.

Harrison, G., Gunnell, D., Glazebrook, C., Page, K., \& Kwiecinski, R. (2001). Association between schizophrenia and social inequality at birth: case-control study. British Journal of Psychiatry, 179, 346-350.

Heinrichs, R. W. (2003). Historical origins of schizophrenia: two early madmen and their illness. Journal of the History of the Behavioural Sciences, 39(4), 349-363.

Insel, T. R., \& Quirion, R. (2005). Psychiatry as a clinical neuroscience discipline. JAMA, 294(17), 2221-2224.

Johnstone, L. (2001). Users and Abusers of Psychiatry (2nd ed.). Hove: Brunner-Routledge.

Light, G., \& Swerdlow, N. (2014). Neurophysiological Biomarkers Informing the Clinical Neuroscience of Schizophrenia: Mismatch Negativity and Prepulse Inhibition of Startle. In V. Kumari, P.Bob \& N.Boutros (eds.) Electrophysiology and Psychophysiology in Psychiatry and Psychopharmacology. New York: Springer

Makari, G. (2008). Revolution in Mind: the creation of psychoanalysis. London: Harper. 
Mathalon, D., \& Ford, J. (2012). Neurobiology of Schizophrenia: Search for the Elusive Correlation with Symptoms, Frontiers in Human Neuroscience 6:136, doi: http://dx.doi.org/10.3389\%2Ffnhum.2012.00136 accessed 18/11/14

Nature Neuroscience. (1999). What causes schizophrenia. Nature Neuroscience, 2, 295.

Noll, R. (2011). The Rise and Fall of Dementia Praecox. Cambridge MA: Harvard University Press.

Papoulias, C., \& Callard, F. (2010). Biology's Gift: interrogating the turn to affect. Body and Society, 16(1), 29-56.

Read, J., Fosse, R., Moskowitz, A., \& Perry, B. (2014). The traumagenic neurodevelopmental model of psychosis revisited. Neuropsychiatry, 4(1), 65-79.

Read, J., van Os, J., Morrison, A. P., \& Ross, C. A. (2005). Childhood trauma, psychosis and schizophrenia: a literature review with theoretical and clinical implications. ActaPsychiatrica-Scandinavica, 112, 330-350.

Roberts, G. (1990). Schizophrenia: the cellular biology of a functional psychosis. Trends in Neurosciences, 13(6), 207-211.

Romme, M., Escher, S., Dillon, J., Corstens, D., \& Morris, M. (2009). Living with Voices: 50 stories of recovery. Ross-on-Wye: PCCS Books.

Rose, H., \& Rose, S. (2012). Genes, Cells and Brains: the promethean promises of the new biology. London: Verso.

Rose, N., \& Abi-Rachid, J. (2013). Neuro. Princeton NJ: Princeton University Press.

Rose, S. (1997). Lifelines: life beyond the gene. Oxford: Oxford University Press.

Tyrka, A. R., Price, L. H., Marsit, C., Walters, O. C., \& Carpenter, L. L. (2012). Childhood adversity and epigenetic modulation of the leukocyte glucocorticoid receptor: preliminary findings in healthy adults. [Research Support, N.I.H., Extramural]. PLoS One, 7(1), e30148.

Woods, A., Jones, N., Bernini, M., Callard, F., Alderson-Day, B., Badcock, J. C., et al. (2014). Interdisciplinary Approaches to the Phenomenology of Auditory Verbal Hallucinations. Schizophrenia Bulletin. 